\title{
Neonatal Pneumonia in NICU of a Tertiary Care Center
}

\section{A MANNAN ${ }^{1}$, NAZMUN NAHAR ${ }^{2}$, FIROZ AHMED ${ }^{3}$, ISMAT JAHAN ${ }^{4}$, TASKINA MOSLEH ${ }^{4}$, KAMRUL AHSAN KHAN ${ }^{5}$, SANJOY KUMER DEY ${ }^{6}$, MOHAMMOD SHAHIDULLAH ${ }^{7}$}

\begin{abstract}
Background: Pneumonia is one of the causes of neonatal infection and responsible for significant morbidity and mortality, especially in developing countries. The study was aimed to reveal frequency and outcome of pneumonia among hospitalized sick newborn of neonatal intensive care unit.

Methodology: This observational study was carried out in the NICU of Bangabandhu Sheikh Mujib Medical University (BSMMU) on 94 neonates with the diagnosis of pneumonia admitted from July 2012 to June 2014. All studied neonates were subjected to history taking, clinical examination, routine investigations, chest radiography and blood culture and sensitivity.

Results: Incidence of neonatal pneumonia was 43\% among admitted neonates with respiratory distress. Mean birth weight and gestational age were $2392 \pm 854$ and $33 \pm 3.9$ weeks respectively. Of enrolled infants with pneumonia, 38 (40.4\%) were early onset, 24 (25\%) were hospital acquired pneumonia and community acquired pneumonia was documented in $14(14.8 \%)$ and the rest $18(19 \%)$ were ventilator associated pneumonia. Blood culture was positive in $18(19 \%)$ of cases with neonatal pneumonia; most common pathogen isolated was acinetobacter. Mean duration of hospital stay was $19 \pm 8$ days. Most of the neonatal pneumonia were cured 72 (76\%) with therapy, whereas 17 (18\%) died during their hospital course.
\end{abstract}

Conclusion: Overall incidence among admitted sick neonates was $8.4 \%$ which constituted $34 \%$ of distressed neonate.

Keywords: Neonatal pneumonia, Ventilator associated pneumonia

Introduction

1. Professor, Department of Neonatology, Bangabandhu Sheikh Mujib Medical University, Dhaka

2. Assistant Professor, Department of Neonatology, $\mathrm{MH}$ Samorita Hospital and Medical College,Tejgaon, Dhaka.

3. Registrar, Department of Paediatrics, Comilla Medical College Hospital,Comilla.

4. Assistant Professor, Department of Neonatology, Bangabandhu Sheikh Mujib Medical University, Dhaka

5. Assistant Professor, Department of Neonatology, Sheikh Shayera Khatun Medical College Hospital, Gopalgonj

6. Associate Professor, Department of Neonatology, Bangabandhu Sheikh Mujib Medical University, Dhaka

7. Professor and Chairman, Department of Neonatology, Bangabandhu Sheikh Mujib Medical University, Dhaka

Correspondence: Dr. M A Mannan, Professor, Department of Neonatology, Room no 219, Block C, Bangabandhu Sheikh Mujib Medical University, Dhaka. Mobile: 01715055506, E-mail: drmannan64@gmail.com

Received: 20 October 2017

Accepted: 25 October 2018
Pneumonia is an inflammatory pulmonary process that may originate in the lung or be a focal complication of a contiguous or systemic inflammatory process. ${ }^{1}$ It is one of the leading fatal childhood disease. ${ }^{2}$ As an important cause of neonatal infection pneumonia accounts for significant morbidity and mortality, with the highest case fatality rate in developing countries. ${ }^{2,3}$ Worldwide neonatal pneumonia is estimated to account for up to $10 \%$ of childhood mortality. ${ }^{2}$ About four million childhood death in developing countries happens due to this fatal disease. ${ }^{4}$

Neonatal pneumonia may be acquired by intrauterine (e.g. transplacental hematogenous, ascending from birth canal), or postnatal routes (e.g. hematogenous, environmental). ${ }^{4,5}$ The pathogens include mainly 
bacteria, followed by viruses and fungi which induce an inflammatory pulmonary condition ${ }^{6}$, causing epithelial injury to the airways. ${ }^{5}$ Early onset pneumonia is mostly caused by ascending infection from maternal genital tract across the membranes, and the baby is often septicemic at birth. Pneumonia of late onset is usually caused by nosocomial infection ${ }^{7}$ especially in mechanically ventilated patients after 48 hours of mechanical ventilation. ${ }^{8}$

Although pneumonia is an important cause of morbidity and mortality among newborn infants, its prompt identification and treatment remain difficult because radiographic changes may be due to atelectasis or noninfectious diseases such as bronchopulmonary dysplasia rather than infection, and infants rarely undergo invasive diagnostic procedures such as bronchoscopy. ${ }^{6}$ There is scarcity of published data regarding burden, clinical features, etiology and outcome of neonatal pneumonia in Bangladesh. The present study was aimed (i)to find the proportion of pneumonia in neonates admitted to a neonatal intensive care unit (ii) evaluate common clinical signs of neonatal pneumonia other than respiratory distress; (iii) determine bacterial etiology of neonatal pneumonia; and (iv) determine outcome of neonatal pneumonia.

\section{Patients and Methods}

This prospective observational study was carried out over a period of two year from July 2012 to June 2014 in the neonatal intensive care unit of Bangabandhu Sheikh Mujib Medical University (BSMMU), Dhaka, Bangladesh. Ninety four neonates with the diagnosis of pneumonia during their hospital stay were enrolled consecutively. Newborn with postnatal age more than 28 days, surgical problem related to the respiratory system and doubtful cases where diagnosis of pneumonia could not be established were excluded.

The baby was evaluated in between feeds and in quiet state. Respiratory rate was recorded for full 1 minute with the help of stop watch in another hand. Pneumonia was diagnosed in the presence of respiratory distress with: (a) Radiologic features suggestive of pneumonia (Presence of any of the following: nodular or coarse patchy infiltrates, diffuse haziness or granularity and lobar or sub-lobar consolidation) with or without Positive blood culture.
Transient episodes of consolidation lasting less than 48 hours due to pulmonary edema were excluded from the diagnosis of neonatal pneumonia.

Respiratory distress is characterized by any of the following: (i) noisy or difficult breathing; (ii) respiratory rate $>60 / \mathrm{min}$; (iii) chest retraction; (iv) cyanosis and (v) grunting. All radiographs suggestive of pneumonia were reviewed by a radiologist who was blinded of clinical findings of enrolled newborns. Then pneumonia was categorized into early onset pneumonia, nosocomial pneumonia, community acquired pneumonia and ventilator associated pneumonia. Pneumonia that became clinically evident within 48 hours of birth was defined as early onset pneumonia. Nosocomial pneumonia was defined as sign symptoms of pneumonia appeared 48 hours after hospital admission. Ventilator associated pneumonia was defined as a nosocomial infection diagnosed in patients undergoing mechanical ventilator for at least 48 hours. Community Acquired pneumonia (CAP) was defined clinically as the presence of signs and symptoms of pneumonia in a previously healthy neonate due to an acute infection (of less than 14 days' duration) which had been acquired in the community outside hospital.

After enrollment all neonates were subjected to clinical assessment by: 1 . History taking, including: patient data, antenatal and perinatal history 2 . Clinical examination 3. Laboratory investigations including complete blood count, C-reactive protein (CRP), blood culture, chest radiography on admission and repeated as required, and arterial blood gases. The babies were followed up till discharge from the hospital or death. Total duration of respiratory therapy and hospital stay were also documented. Data analysis was performed using SPSS version 20 (SPSS, Chicago, IL). The analysis of patient demographics and baseline outcome variables were summarized using descriptive summary measures: expressed as mean for numerical variables and percent for categorical variables.

\section{Results}

During the study period total number of admissions was 1108; respiratory distress was the presenting feature in $271(24 \%)$ cases. Of the 271 cases of respiratory distress, pneumonia was the underlying 
cause in 94 babies; resulting in an incidence of neonatal pneumonia as $34 \%$ among the baby with respiratory distress. Overall incidence among admitted sick neonates was $8.4 \%, 94 / 1108$.

Of enrolled infants with pneumonia, $38(40.4 \%)$ were early onset pneumonia, rest $56(55.6 \mathrm{l} \%)$ were late onset pneumonia. Among late onset pneumonia 32/ $56(57 \%)$ were presented as hospital acquired pneumonia and community acquired pneumonia was documented in $24 / 56(43 \%)$. Ventilator associated pneumonia were 18/94 (19\%) .

Among neonatal pneumonia case, 59 (62.7\%) were male and 52(55\%) were out born. Mean birth weight and gestational age were $2392 \pm 854$ grams and $33 \pm 3.9$ weeks respectively shown in table 1 . LUCS was the predominant mode of delivery in two-thirds of enrolled patients. Distribution of pneumonia was higher among outborn delivery ( $55 \%$ vs $45 \%$ ). Among 38 early onset pneumonia, majority $30(79 \%)$ had documented maternal risk factor for early onset sepsis (Table-I). Table-Il shows potential risk factors for early onset pneumonia. Among them prolonged rupture of membrane was documented in majority of the enrolled newborn $25 / 30(83 \%)$.

Table-I

Demographic variables of enrolled infants

\begin{tabular}{lc}
\hline Variable & Infants $(n=94)$ \\
\hline Sex & \\
$\quad$ Male & $59(62.7 \%)$ \\
$\quad$ Female & $35(37.2 \%)$ \\
Place of Delivery & \\
$\quad$ Outborn & $52(55.4 \%)$ \\
$\quad$ Inborn & $42(44.6 \%)$ \\
Mode Of Delivery & \\
$\quad$ Vaginal & $30(32 \%)$ \\
$\quad$ LUCS & $64(68 \%)$ \\
Birth weight (Mean \pm SD) & $2392 \pm 854$ \\
Gestational Age (Mean \pm SD) & $33 \pm 3.9$ \\
Maternal risk factor for EOP (n 38) & \\
$\quad$ Yes & $30(79 \%)$ \\
$\quad$ No & $8(21 \%)$ \\
\hline
\end{tabular}

EOP Early Onset Pneumonia

Numerical data are presented as mean $\pm \mathrm{sd}$ and categorical data as percentage (\%)
Table-II

Risk factor for early onset pneumonia among studied infant

\begin{tabular}{lc}
\hline Variable & Infants $(\mathrm{n}=30)$ \\
\hline Prolonged ruptured of membrane & 25 \\
(>18hours) & \\
Maternal fever $\left(>38^{\circ} \mathrm{F}\right)$ & 6 \\
Foul smelling liquor & 2 \\
Repeated per vaginal examination & 2 \\
\hline
\end{tabular}

Figure 1 shows common presentation of newborn with neonatal pneumonia. Tachypnoea was the commonest respiratory manifestation. Next to tachypnoea, chest retraction and nasal flaring were also commonly seen among pneumonic patients. Apart from respiratory presentation, lethargy was the commonest nonspecific presenting feature of neonatal pneumonia.

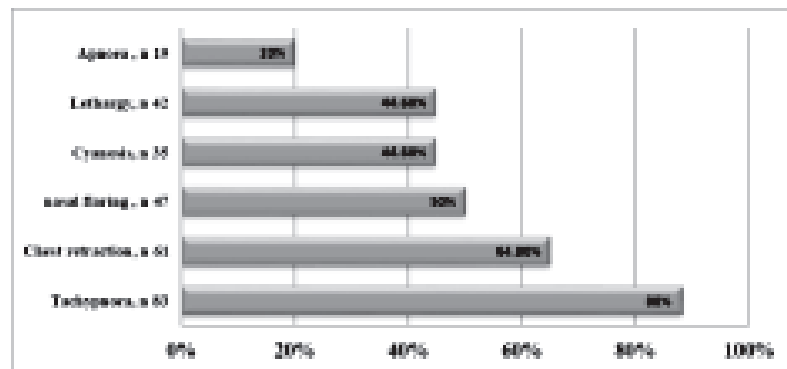

Fig.-1: Presenting manifestation of neonatal pneumonia

Sepsis screening (hematologic parameters and CRP) were suggestive in $49(52.1 \%)$ of enrolled cases. Blood culture was positive in $18(19 \%)$ out of 94 cases of neonatal pneumonia; the pathogen isolates are shown in table III.

Table-III

Bacterial pathogen isolation from enrolled newborn with pneumonia

\begin{tabular}{lc}
\hline Name of the pathogen & Infants $(\mathrm{n}=18)$ \\
\hline Acinetobacter & 9 \\
E. Coli & 4 \\
Klebsiella spp. & 3 \\
Pseudomonas & 1 \\
Enterobacter & 1 \\
\hline
\end{tabular}


Most common radiologic change was localized/ diffuse alveolar infiltrates in more than half 58\% (55/ 94 ) of the newborn with pneumonia. Other findings were uni and or bilateral haziness and sublobar, lobar consolidation in 23\% (22/94) and 18\% (17/94) and respectively (Figure 2 and 3 ).

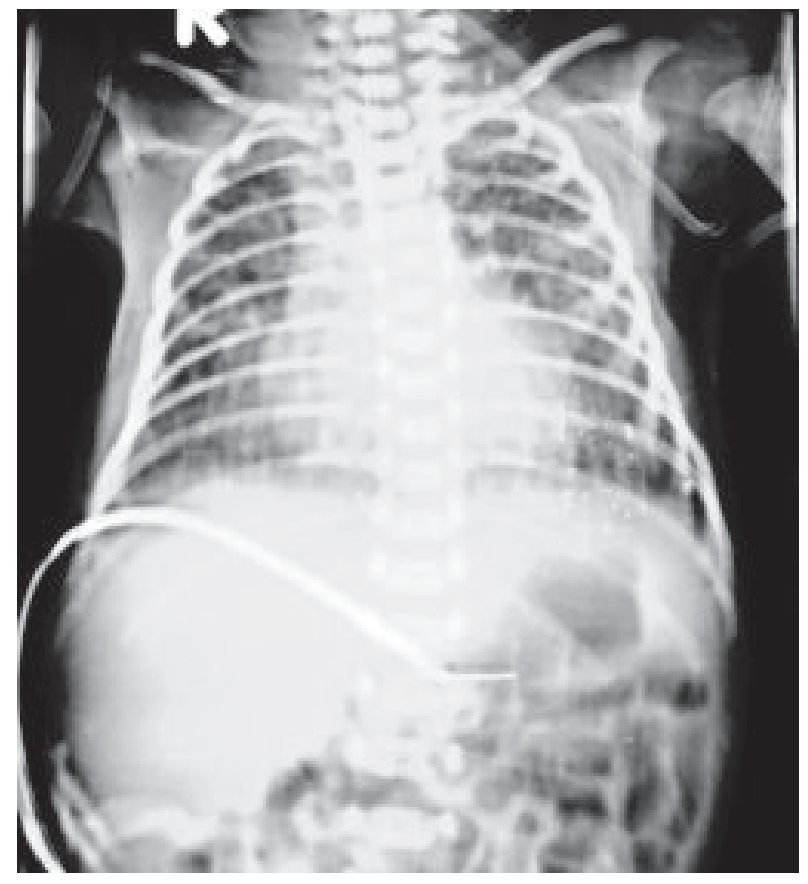

Fig.-2: Bilateral diffuse alveolar infiltration in an enrolled preterm newborn presenting early onset pneumonia

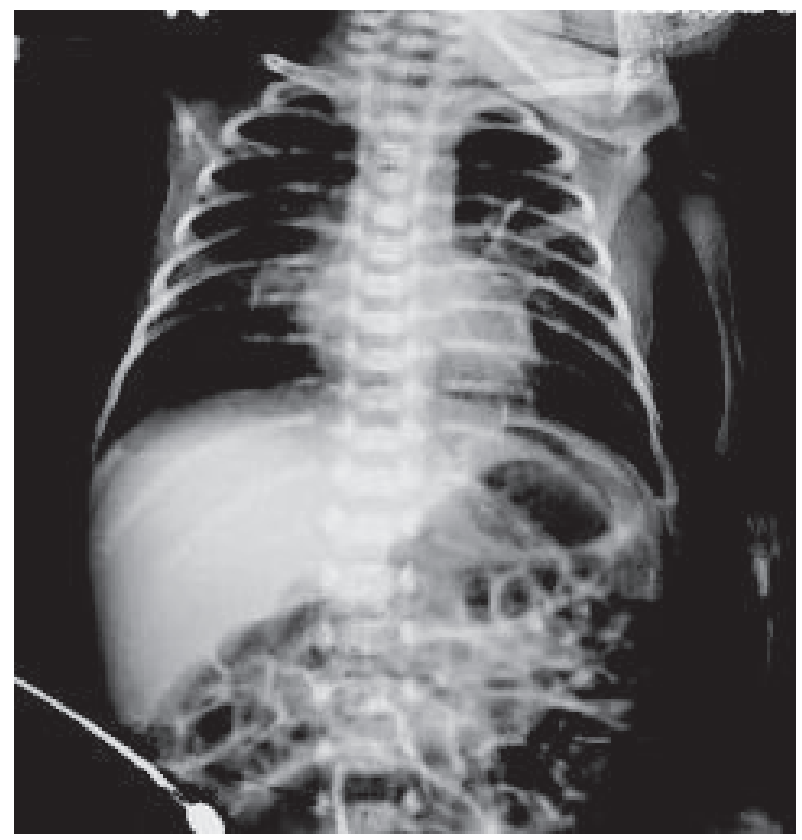

Fig.-3: Inhomogenous opacity in an enrolled preterm newborn presenting early onset pneumonia
Mean duration of hospital stay was $13 \pm 8$ and $19 \pm 8$ days. Majority $76.5 \%$ (72/94) of the neonatal pneumonia were cured while 5\% (5/94) left against medical advice and $18 \%$ (17/94) died during their hospital course (Figure 2).

\section{Discussion}

In this study, a total of 94 neonatal pneumonia cases were included over two year's duration, mean birth weight and gestational age was $2392 \pm 854$ grams and $33 \pm 3.9$ weeks respectively. In this study, the mean birth weight of pneumonia cases was lower which was similar to the result obtained by Khattab et al. ${ }^{11}$ Also lower mean gestational age of infants with pneumonia were documented which was in agreement with other studies enrolled ventilator associated pneumonia cases only. ${ }^{11}$

Overall incidence of pneumonia among admitted neonate was $8.4 \%$. Of infants with respiratory distress, incidence of pneumonia was $34 \%$. The variable incidences were demonstrated by the earlier studies ranging from $18.7 \%$ to $68.7 \% .{ }^{12-16} \mathrm{~A}$ study from India showed higher incidence $(68.7 \%)$ in comparison to other reports. ${ }^{17}$

While earlier studies ${ }^{12-16}$ exclusively enrolled inborn with respiratory distress, we aimed to figure out the overall incidence among both inborn and outborn. In this study, the distribution of pneumonia was slightly higher among outborn delivery ( $55 \%$ vs $45 \%$ ).

The overall mortality rate in the present study was $18 \%$ which is lower than that reported from other studies. ${ }^{12,13,17,18}$ Wide range of mortality rate from $8 \%$ up to $48 \%$ was reported in a review of relevant studies done in developing countries ${ }^{2}$. Eight percent death was reported in a study done in the emergency department of a referral centre of India ${ }^{19}$.

In this study, near about half $(40.4 \%)$ of the enrolled neonates were having early onset pneumonia. It is important to recognize, however, that risk factors are often absent in babies who develop pneumonia of early onset and prolonged rupture of membrane $(>18$ hours) were documented in most of the cases $(85 \%)$ of early onset pneumonia which was comparable with the study done by Webber $\mathrm{S}$ et al. ${ }^{7}$ We have not been able to show clearly the etiological agent in those babies who we thought had pneumonia but who had negative blood cultures. Our findings support the practice of many units that all neonates with respiratory distress should be given antibiotics 
intravenously for at least 48 to 72 hours until the results of blood cultures are available.

In the current study, bacterial etiology of pneumonia was established only in $18(19 \%)$ out of 94 cases; the yield is low in comparison to other studies. ${ }^{17,18,20}$ The most common pathogen isolated was acinetobacter ( 9 cases) followed by E coli ( 5 cases) and Klebsiella (4 cases). Acinetobcter was very infrequent findings in earlier studies done in Indian subcontinent ${ }^{2}$. Increasing incidence of Klebsiella isolation was observed in several studies done in developing countries on neonatal pneumonia. ${ }^{20-23}$

\section{Conclusion}

Overall incidence of pneumonia among admitted sick neonates was $8.4 \%$ which constitutes $34 \%$ of distressed neonate. Acinetobacter was the common organism isolated from enrolled pneumonia cases. Majority of the neonatal pneumonia were cured while $18 \%$ died during their hospital course.

\section{Limitation}

There is no universally accepted definition of pneumonia in the neonatal period. Lack of a clearcut definition patient enrollment difficult and conclusions may differ from one study to another. Another limitation is the small sample size of this study.

\section{Acknowledgement:}

We gratefully acknowledge the sincere cooperation and contribution of all doctors, nurses, staff of the department of Neonatology, Bangabandhu Sheikh Mujib Medical University (BSMMU). We would like to convey our gratitude to mothers and care givers of the study newborns for their consent to enroll their babies in this study.

\section{References}

1. Muhammad Aslam, Congenital Pneumonia, http://emedicine.medscape.com/article/ 978865-overview. Last update-03.01.2016.

2. Duke T. Neonatal pneumonia in developing countries. Arch Dis Child Fetal Neonatal Ed 2005; 90:F211 Arch Dis Child Fetal Neonatal Ed 2005;90:F211-FF219

3. Black RE, Cousens S, Johnson HL et al. Global, regional and national causes of child mortality in 2008: a systematic analysis, Lancet 2010;375:1969-87
4. Bang AT, Bang R A, Morankar V P, Sontakke P $G$, Solanki J M. Pneumonia in neonates: can it be managed in the community? Archives disease in childhood 1993;68:550-556.

5. Reiterer F. Neonatal Pneumonia. http:// www.intechopen.com/books/neonatal-bacterialinfection/neonatal pneumonia. Published-April 30,2013

6. Polin RA, Saiman L. Nosocomial infections in the neonatal intensive care unit. NeoReviews 2003;4(3) : 81-88.

7. Webber S,Wilkinson AR, Lindsell D, Hope PL, Dobson SRM, Isaacs D. Neonatal pneumonia. Archives disease in childhood 1990;65:207-211.

8. Tripathi S, Malik GK,Jain A, Koheli N. Study of ventilator associated pneumonia in neonatal intensive care unit:characteristics, risk factors and outcome. Internal journal of medical update 2010;5(1) :12-19.

9. Stoll BJ. The global impact of neonatal infection. Clin Perinatol 1997; 24:1.

10. Garenne M, Ronsmans C, Campbell H. The magnitude of mortality from acute respiratory infections in children under 5 years in developing countries. World Health Stat Q 1992; 45:180.

11. KhattabAA, El-Lahony DM, Soliman WF. Ventilatorassociated pneumonia in the neonatal intensive care unit. Menoufia Med J 2014;27:73-7

12. Khatua SP, Gangwal A, Basu P, Patodhi PKR. The incidence and etiology of respiratory distress in newborn. Indian Pediatr 1979; 16: 1121-1126.

13. Thomas S, Verma IC, Singh M, Menon PSN. Spectrum of respiratory distress syndrome in North India. A prospective study. Indian J Pediatr 1981; 48: 61-65.

14. Misra PK. Respiratory distress in newborn. Indian Pediatr 1987; 24: 77-80.

15. Kumar P, Kumar R, Narang A. Spectrum of neonatal respiratory distress at PGI. Bull NNF 1999; 13: 8-11.

16. Jhalmarson O. Epidemiology and classification of acute neonatal respiratory disorders. Acta Pediatr Scand 1981; 70: 773-783. 
17. Mathur N B, Garg K and Kumar S. Respiratory distress in neonates with special reference to Pneumonia. Indian Paediatr 2002; 39: 529-37.

18. Shakunthala SKV, Rao GM, Urmila S. Diagnostic lung puncture aspiration in acute pneumonia of newborn. Indian Pediatr 1978; 15: 39-44.

19. Singhi S, Singhi PD. Clinical signs in neonatal pneumonia. Lancet 1990; 336: 1072-73.

20. Mishra S, Bhakoo ON, Ayyagiri A, Katariya S. Clinical and bacteriological profile of neonatal pneumonia. Indian J Med Res 1991; 93: 366370.

21. Bhakoo ON. Neonatal bacterial infections at Chandigarh: A decade of experience. Indian J Pediatr 1980; 47: 419-424.

22. Singh M. Perinatal infection. In: Care of the Newborn, Delhi, 4th edn. Sagar Publications, New Delhi 1991; pp 154-176.

23. Mishra JN, Rai MG, Chakravarty S, Prasad S. Study of neonatal septicemia. Indian Pediatr 1985; 22: 281-285. 